\title{
Coupling Impedances for Perforated Beam Pipes with General Shape from Impedance Boundary Condition
}

\author{
Stefania Petracca*
}

\begin{abstract}
We introduce an equivalent wall impedance to describe the electromagnetic boundary conditions at perforated pipe walls. The new impedance boundary condition, together with our general formulae for computing longitudinal and transverse beam coupling impedances in complex heterogeneous pipes, provides a good trade-off between computational accuracy and ease.
\end{abstract}

Geneva, Switzerland

February 3, 1999

On leave of absence from Universita' del Sannio, Benevento and I.N.F.N. Salerno, Italy. 


\section{Introduction}

In the foreseen LHC design [1], the stainless steel vacuum chamber (cold bore) will be kept at $1.9 \mathrm{~K}$ using superfluid Helium, and protected from synchrotron radiation by a beam screen cooled at some 4.5 to $20 \mathrm{~K}$. Gas desorption due to synchrotron radiation, and subsequent surface deposition limits the pumping efficiency of the beam pipe vacuum system, unless many holes or slots are drilled in the beam screen wall, allowing for a transfer of the excess gas to the $1.9 \mathrm{~K}$ cold bore, where the pumping capacity is adequate. At the present stage of the project, the total number of holes or slots should be as large as $10^{7}-10^{8}$ $\left(10^{2}-10^{3}\right.$ holes/slots per meter), with typical sizes of $\sim 2 \mathrm{~mm}$ diameter (holes) or $1.5 \times 8 \mathrm{~mm}$ (slots). The effect of so many holes/slots on the beam dynamics and stability, e.g. in terms of coupling impedances, is a fundamental issue and has been carefully investigated, both theoretically [2]-[6], and experimentally [7], [8].

In this paper we introduce a new (local) impedance boundary condition (b.c.), of the Leontóvich type [9], to describe perforated pipe walls. The latter can be used within the general framework presented in [10] and summarized in Sect. 2 to obtain analytic estimates, based on reciprocity formulae, of the longitudinal and transverse coupling impedances for heterogenous beam pipes with complex geometry. The coupling impedances of a simple, unperturbed pipe assumed known, can be related to those of another pipe differing from the former by some perturbation in the boundary geometry and/or constitutive properties. The involved computations are straightforward (geometric and constitutive effects are embodied in separate factors) and reasonably accurate.

In the usual approach, based on the first order approximation of Bethe's theory [11], computation of the coupling impedances due to the presence of the holes in the pipe wall requires modal expansion of the field in the pipe geometry. This is possible only for geometries where the Helmoltz (potential) equation is separable. On the other hand, our (perturbative) approach (reciprocity formulae + impedance boundary condition + Bethe approximation) introduces an equivalent wall impedance to describe the electromagnetic boundary conditions at a perforated pipe wall. Therefore its advantage lies in the possibility of considering transverse pipe geometries where the modal analysis cannot be performed.

In Sect. 3 we introduce an impedance boundary condition appropriate to a thin perfectly conducting pipe wall with many (non interacting) electrically small holes in free space starting from a heuristic argument. In Sect. 4 we derive the same result by solving a rigorous boundary value problem. Possible model improvements are considered in Sect. 5, including: (i) holes in a thick wall, (ii) interacting holes, and (iii) perforated beam pipes in a coaxial lossy tube. In Sect. 6 we apply our formalism to a possible $L H C$ geometry and compare our results to those obtained by other Authors using a different approach, while in Sect. 7 we compute the parasitic loss. Conclusions follow under Sect. 8. Relevant tools 


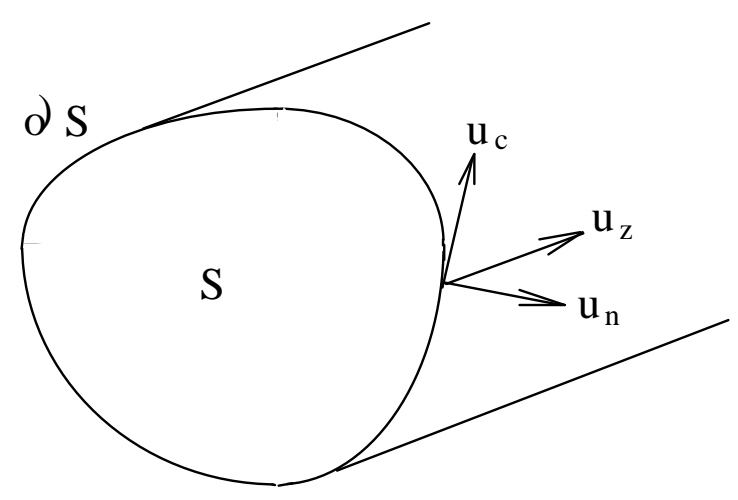

Figure 1: Unit vectors $\hat{u}_{c}, \hat{u}_{n}, \hat{u}_{z}$ relevant to eqs. (1) and (2).

and definitions are collected in Appendix $A$ to $D$.

\section{Coupling Impedances in Complex Pipes}

According to [10] the specific longitudinal and transverse beam coupling impedances $Z_{0, \|}(\omega)$ and $\bar{Z}_{0, \perp}(\omega)$ of a simple, unperturbed pipe (e.g., circular, perfectly conducting) assumed known, can be related to those $Z_{\|}(\omega), \bar{Z}_{\perp}(\omega)$ of another pipe differing from the former by some perturbation in the boundary geometry and/or constitutive properties, as follows:

$$
\begin{gathered}
Z_{\|}(\omega)-Z_{0, \|}(\omega)=\frac{\epsilon_{0}}{\beta_{0} c Q^{2}}\left\{Y_{0} \oint_{\partial S} Z_{\text {wall }} E_{0 n}^{(i r r .) *}(\vec{r}, 0) .\right. \\
\left.\left[\beta_{0} E_{n}^{(i r r .)}(\vec{r}, 0)+\beta_{0}^{-1} E_{n}^{(s o l .)}(\vec{r}, 0)\right] d \ell-\oint_{\partial S} E_{0 z}^{*}(\vec{r}, 0) E_{n}^{(i r r .)}(\vec{r}, 0) d \ell\right\}, \\
\bar{Z}_{\perp}(\omega)-\overline{\bar{Z}}_{0, \perp}(\omega)=\frac{\epsilon_{0}}{\beta_{0} c Q^{2} k}\left\{Y_{0} \oint_{\partial S} Z_{\text {wall }} \nabla_{\vec{r}_{0}} E_{0 n}^{(i r r .) *}\left(\vec{r}, \vec{r}_{0}\right) \otimes\right. \\
\otimes \nabla_{\vec{r}_{1}}\left[\beta_{0} E_{n}^{(i r r .)}\left(\vec{r}, \vec{r}_{1}\right)+\beta_{0}^{-1} E_{n}^{(s o l .)}\left(\vec{r}, \vec{r}_{1}\right)\right] d \ell+ \\
\left.-\oint_{\partial S} \nabla_{\vec{r}_{0}} E_{0 z}^{*}\left(\vec{r}, \vec{r}_{0}\right) \otimes \nabla_{\vec{r}_{1}} E_{n}^{(i r r .)}(\vec{r}, 1) d \ell\right\}_{\vec{r}_{1}=\vec{r}_{0}=0},
\end{gathered}
$$

where $c=\left(\epsilon_{0} \mu_{0}\right)^{-1 / 2}$ is the speed of light in vacuum, $Y_{0}=\left(\epsilon_{0} / \mu_{0}\right)^{1 / 2}$ is the vacuum characteristic admittance, $\epsilon_{0}$ and $\mu_{0}$ being the vacuum permittivity and permeability, $\beta_{0}$ is the relativistic factor, $Q$ is the total beam charge, $\vec{E}_{0}^{(\text {sol. })}, \vec{E}_{0}^{(i r r .)}$ are the solenoidal and irrotational parts of the electric field in the unperturbed pipe, the unit vectors $\hat{u}_{c}, \hat{u}_{n}, \hat{u}_{z}$ are defined in Fig. $1, \overline{\bar{Z}}_{\perp}(\omega)$ is a tensor and one assumes an impedance (Leontóvich) boundary condition to hold at the pipe wall $\partial S:$ 


$$
\left|\left(\overline{\bar{I}}-\hat{u}_{n} \hat{u}_{n}\right) \cdot \vec{E}-Z_{\text {wall }} \hat{u}_{n} \times \vec{H}\right|_{\partial S}=0
$$

where $Z_{\text {wall }}$ is the pipe-wall complex characteristic impedance.

The first integral term on the r.h.s of (1) accounts for the effect of the finite wall conductivity, and is nonzero if and only if $Z_{\text {wall }}$ is not identically zero on $\partial S$. The second integral on the r.h.s. of (1), on the other hand, accounts for the effect of the geometrical perturbation of the boundary, and is non-zero if and only if the unperturbed axial field component $E_{0 z}$ is not identically zero on $\partial S^{1}$.

For the simplest case of a circular pipe with radius $b$ and uniform wall impedance $Z_{\text {wall }}$ one readily obtains from eq. (1):

$$
Z_{\|}=\frac{Z_{\text {wall }}}{2 \pi b}, \quad \overline{\bar{Z}}_{\perp}=\frac{Z_{\text {wall }}}{\pi k_{0} b^{3}}\left(\hat{u}_{x} \hat{u}_{x}+\hat{u}_{y} \hat{u}_{y}\right) .
$$

in agreement with the known exact result [12].

\section{Impedance Boundary Conditions at Perforated Beam Pipe Wall: Heuristics}

Extensive calculations and estimates for the longitudinal and transverse impedances per unit length in perforated beam pipes have been performed by S. Kurennoy ${ }^{2}$ and R. Gluckstern [2]-[6].

According to these Authors, the longitudinal impedance per unit length of a circular beam pipe with radius $b$ carrying $N_{\lambda}$ holes per unit length is ${ }^{3}$ :

$$
Z_{\|}=-j Z_{0} k_{0} \frac{\left(\alpha_{e}+\alpha_{m}\right)}{4 \pi^{2} b^{2}} N_{\lambda},
$$

where $k_{0}=\omega / c$ is the free-space wavenumber, $c$ the light velocity in vacuum, and $\alpha_{e, m}$ the electric and magnetic polarizabilities of each hole ${ }^{4}$. The result $(5)$ is independent of the azimuthal position of the holes, such being the field produced by an axial beam in a circular pipe. Thus, letting:

$$
n_{\sigma}=\frac{N_{\lambda}}{2 \pi b}
$$

represent the number of holes per unit wall area, eq. (5) can be written:

\footnotetext{
${ }^{1}$ The beam impedances are obviously independent of the total beam charge, as the field in (1), (2) are proportional to $Q$.

${ }^{2} \mathrm{~A}$ notable result of Kurennoy's analysis is related to the occurrence of trapped modes in the neighbourhood of pipe discontinuities, including holes [13].

${ }^{3}$ Kurennoy has also computed the (negligible) real correction to (5) resulting from radiation leakage through the holes [3].

${ }^{4}$ Polarizabilities for most hole shapes can be found in [14]-[16].
} 


$$
Z_{\|}=-j Z_{0} k_{0} \frac{\left(\alpha_{e}+\alpha_{m}\right)}{2 \pi b} n_{\sigma}
$$

By comparing eq.s (7) and (4), one is led to the heuristic conclusion that a perforated wall could be described by an impedance boundary condition with:

$$
Z_{\text {wall }}=-j Z_{0} k_{0}\left(\alpha_{e}+\alpha_{m}\right) n_{\sigma} .
$$

Using (8) in (1) and (2) would allow to estimate the longitudinal and transverse beam impedances under very general assumptions, including e.g. pipes with unevenly distributed holes, and complicated transverse geometries. This is indeed the case $^{5}$, as can be seen by considering a pipe with general transverse geometry carrying $N_{\lambda}$ (uniformly spaced) holes per unit length, located at $\ell=\ell_{h}, \ell$ being the arc-length along the pipe cross-section contour $\partial S$, for which:

$$
n_{\sigma}=N_{\lambda} \delta\left(\ell-\ell_{h}\right)
$$

Using (9) in (1), gives

$$
Z_{\|}=-j Z_{0} k_{0}\left(\alpha_{e}+\alpha_{m}\right) e_{n}\left(\ell_{h}\right) e_{n}^{*}\left(\ell_{h}\right),
$$

where $e_{n}\left(\ell_{h}\right)=\left(Q / \epsilon_{0}\right)^{-1} E_{n}\left(\ell_{h}\right), E_{n}\left(\ell_{h}\right)$ being the normal electric field at the hole position produced by an axial beam with total charge $Q$. Equation (10) reproduces exactly Kurennoy's result valid for this most general case [17].

In the next section we shall further support the heuristic result (8) by solving a rigorous EM boundary value problem.

\section{Impedance Boundary Conditions at Perforated Beam Pipe Wall: Boundary Value Approach}

In this section we consider a $T M$ plane wave $\left(\vec{E}_{i}, \vec{H}_{i}\right)$ :

$$
\begin{aligned}
& \vec{k}^{(i)}=k_{0}\left(\sin \theta \hat{u}_{x}+\cos \theta \hat{u}_{z}\right), \\
& \vec{H}^{(i)}=H_{0} \hat{u}_{y} e^{j \vec{k}^{(i)} \cdot \vec{r}}=H_{0} \hat{u}_{y} e^{-j k_{0}(z \cos \theta+x \sin \theta)}, \\
& \vec{E}^{(i)}=Z_{0} \vec{H}^{(i)} \times \vec{k}^{(i)}=\left(-\sin \theta \hat{u}_{z}+\cos \theta \hat{u}_{x}\right) Z_{0} H_{0} e^{j \vec{k}^{(i)} \cdot \vec{r}},
\end{aligned}
$$

incident with an angle $\theta$ on a perfectly conducting plane at $z=0$ bearing a regular array of holes at $x=n a_{h}, y=m b_{h}, m, n=-\infty, \ldots, \infty$ (see Fig. 2). In (11) $Z_{0}=\left(\mu_{0} / \epsilon_{0}\right)^{1 / 2}$ is the free space impedance. Note that we use the $\exp (-j \omega t)$ time dependance (which is rather unusual in electromagnetics), to comply with the Particle Accelerator Literature.

\footnotetext{
${ }^{5}$ The only restrictive underlying assumption is that the holes should be (piecewise) uniformly-distributed in the longitudinal direction, which is needed for the very definition of beam impedance per unit length to apply.
} 


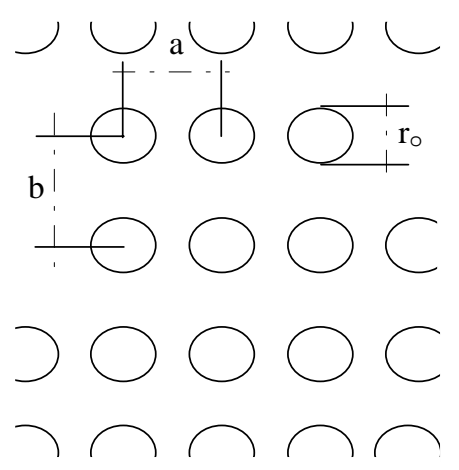

Figure 2: Regular 2D array of circular holes of radius $r_{0}$ on a conducting plane at $x=n a_{h}, y=m b_{h}, m, n=-\infty, \ldots, \infty$.

In the limit of near-grazing incidence $\theta \longrightarrow \pi / 2$ the primary field (holes suppressed) has the same local structure at $z=0$ as the field of a relativistic particle beam at a perfectly conducting pipe wall: the magnetic field is nearlytangent to the wall, and the electric field nearly normal. Thus, following [18] we expect to be able to deduce an appropriate (local) impedance boundary condition from the asymptotic $(\theta \longrightarrow \pi / 2)$ form of the $T M$ plane wave reflection coefficient.

If the holes were absent the reflected field $\left(\vec{E}^{(r)}, \vec{H}^{(r)}\right)$ would be:

$$
\begin{aligned}
& \vec{k}^{(r)}=k_{0}\left(\sin \theta \hat{u}_{x}-\cos \theta \hat{u}_{z}\right), \\
& \vec{H}^{(r)}=H_{0} \hat{u}_{y} e^{j \vec{k}^{(r)} \cdot \vec{r}}=H_{0} \hat{u}_{y} e^{j k_{0}(-z \cos \theta+x \sin \theta)}, \\
& \vec{E}^{(r)}=Z_{0} \vec{H}^{(r)} \times \vec{k}^{(r)}=\left(-\sin \theta \hat{u}_{z}-\cos \theta \hat{u}_{x}\right) Z_{0} H_{0} e^{j \vec{k}^{(r)} \cdot \vec{r}},
\end{aligned}
$$

and the total field:

$$
\left(\vec{E}_{t o t}, \vec{H}_{t o t}\right)= \begin{cases}0, & z>0 \\ \left(\vec{E}^{(i)}+\vec{E}^{(r)}, \vec{H}^{(i)}+\vec{H}^{(r)}\right), & z<0 .\end{cases}
$$

The field in the presence of the holes, according to Bethe's approximation [11], can be computed by adding to the primary field (13) above, the field $\vec{E}^{(\text {scatt. })}, \vec{H}^{\text {(scatt. })}$ produced by elementary electric and magnetic sources radiating on a perfectly conducting (no hole) plane $z=0$ and placed at $x=n a_{h}, y=m b_{h}$, $m, n=-\infty, \ldots, \infty$.

Specifically, the sources at $\vec{r}=\vec{r}_{n m}=n a_{h} \hat{u}_{x}+m b_{h} \hat{u}_{y}$, have moment densities:

$$
\begin{aligned}
& \vec{P}_{n m}=\epsilon_{0} \alpha_{e} \delta\left(\vec{r}-\vec{r}_{m n}\right) E_{z}^{(t o t)}\left(\vec{r}_{m n}\right) \hat{u}_{z}, \\
& \vec{M}_{n m}=\alpha_{m} \delta\left(\vec{r}-\vec{r}_{m n}\right)\left(\overline{\bar{I}}-\hat{u}_{z} \hat{u}_{z}\right) \vec{H}^{(t o t)}\left(\vec{r}_{m n}\right),
\end{aligned}
$$

where and $\alpha_{e}$ and $\alpha_{m}$ are the hole electric and magnetic polarizabilities ${ }^{6}$. It is seen from eq.s (13), (11) and (12) that:

\footnotetext{
${ }^{6}$ Since we are interested in computing the hole-produced field in $z<0$, we use the so called internal hole polarizabilities in (14).
} 


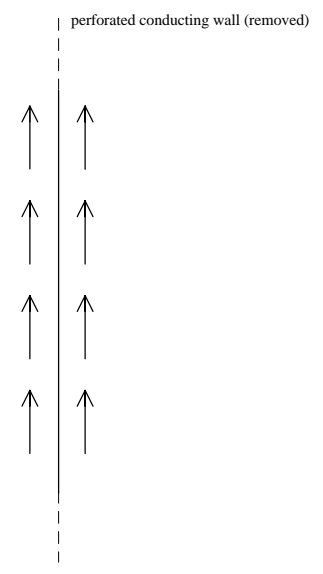

Figure 3: Equiverse magnetic Bethe-dipoles images.

$$
\begin{aligned}
& E_{z}^{(t o t)}(z=0)=2 E_{z}^{(i)}(z=0)=-Z_{0} H_{0} \sin \theta e^{j k_{0} x \sin \theta} \\
& \left(\overline{\bar{I}}-\hat{u}_{z} \hat{u}_{z}\right) \cdot \vec{H}^{(t o t)}(z=0)=\left(\overline{\bar{I}}-\hat{u}_{z} \hat{u}_{z}\right) \cdot 2 \vec{H}^{(i)}(z=0)=2 H_{0} \hat{u}_{y} e^{j k_{0} x \sin \theta} .
\end{aligned}
$$

Furthermore, the fields radiated in $z<0$ by the dipoles (14) sitting on the perfectly conducting plane $z=0$ are the same as those radiated in free space by (14) and their images ${ }^{7}$. The images are equiverse and placed exactly at the same positions as the corresponding primary sources (see Fig. 3).

Thus the superposition of the primary and image sources is just twice (14), viz.:

$$
\begin{gathered}
\vec{P}_{t o t}=\sum_{m, n}\left(\vec{P}_{m, n}+\vec{P}_{m, n}^{(i m a g e)}\right)=\sum_{m, n} 2 \vec{P}_{m, n}= \\
=-4 c^{-1} H_{0} \alpha_{e} \sin \theta \delta(z) \sum_{m, n} \delta\left(x-n a_{h}\right) \delta\left(y-m b_{h}\right) e^{j k_{0} n a_{h} \sin \theta} \hat{u}_{z}, \\
\vec{M}_{t o t}=\sum_{m, n}\left(\vec{M}_{m, n}+\vec{M}_{m, n}^{(i m a g e)}\right)=\sum_{m, n} 2 \vec{M}_{m, n}= \\
=4 H_{0} \alpha_{m} \delta(z) \sum_{m, n} \delta\left(x-n a_{h}\right) \delta\left(y-m b_{h}\right) e^{j k_{0} n a_{h} \sin \theta} \hat{u}_{y} .
\end{gathered}
$$

The fields produced by these sources can be quickly computed using the vector potential $\vec{A}$ and the magnetic Hertz potential $\vec{\Pi}$ which are related to $\vec{P}_{t o t}$ and $\vec{M}_{\text {tot }}$ by:

${ }^{7}$ The field computed in this way fails to reproduce the true field for $z>0$. If one were interested in computing the fields in $z>0$, one should use the external polarizabilities in (14) before applying the image theorem. In this case, the computed field would not reproduce the true field for $z<0$. 


$$
\begin{aligned}
& \left(\nabla^{2}+k_{0}^{2}\right) \vec{A}=j \omega \mu_{0} \vec{P}_{t o t} \\
& \left(\nabla^{2}+k_{0}^{2}\right) \vec{\Pi}=-\vec{M}_{t o t}
\end{aligned}
$$

as follows:

$$
\begin{aligned}
& \vec{E}^{(\text {scatt. })}=-j \omega c^{-2} \nabla \times \nabla \times \vec{A}+j \omega \mu_{0} \nabla \times \vec{\Pi}, \\
& \vec{H}^{(\text {scatt. })}=\mu_{0}^{-1} \nabla \times \vec{A}+\nabla \times \nabla \times \vec{\Pi} .
\end{aligned}
$$

In order to solve eq.s (18) it is first expedient to note that since $\vec{P}_{\text {tot }}=P \hat{u}_{z}$ and $\vec{M}_{\text {tot }}=M \hat{u}_{y}$ then $\vec{A}=A \hat{u}_{z}$ and $\vec{\Pi}=\Pi \hat{u}_{y}$. It is further convenient to use the (generalized) Fourier representation of the periodic delta functions:

$$
\sum_{n} \delta\left(x-n a_{h}\right)=\frac{1}{a_{h}} \sum_{p} e^{j q\left(2 \pi / a_{h}\right) x}, \quad \sum_{m} \delta\left(y-m b_{h}\right)=\frac{1}{a_{h}} \sum_{q} e^{j q\left(2 \pi / b_{h}\right) y},
$$

so as to recast the source terms into the following form:

$$
\begin{aligned}
& \vec{P}_{t o t}=-\frac{4 H_{0} \alpha_{e}}{c b_{h} a_{h}} \sin \theta \delta(z) \sum_{p, q} e^{2 \pi j\left(p x / a_{h}+q y / b_{h}\right)} e^{j k_{0} x \sin \theta} \hat{u}_{z}, \\
& \vec{M}_{t o t}=\frac{4 H_{0} \alpha_{m}}{b_{h} a_{h}} \delta(z) \sum_{p, q} e^{2 \pi j\left(p x / a_{h}+q y / b_{h}\right)} e^{j k_{0} x \sin \theta} \hat{u}_{y},
\end{aligned}
$$

and obtaining the following wave-equations:

$$
\begin{aligned}
& \left(\nabla^{2}+k_{0}^{2}\right) A=-j \omega \mu_{0} \frac{4 H_{0} \alpha_{e}}{c a_{h} b_{h}} \sin \theta \delta(z) \sum_{p, q} e^{2 \pi j\left(p x / a_{h}+q y / b_{h}\right)} e^{j k_{0} x \sin \theta}, \\
& \left(\nabla^{2}+k_{0}^{2}\right) \Pi=-\frac{4 H_{0} \alpha_{m}}{a_{h} b_{h}} \delta(z) \sum_{p, q} e^{2 \pi j(p x / a+q y / b)} e^{j k_{0} x \sin \theta} .
\end{aligned}
$$

The forcing terms in (22) contain delta functions at $z=0$, and thus the equations must be solved in the weak (Sobolev) sense.

The form of the equations suggests that the solutions have the same $x, y$ dependance as the corresponding forcing terms, and thus can be written as:

$$
\begin{aligned}
& A=\sum_{p, q} A_{p, q} e^{ \pm j \gamma_{p, q} z} e^{j\left[k_{0} x \sin \theta+2 \pi\left(p x / a_{h}+q y / b_{h}\right)\right]}, \quad z \gtrless 0, \\
& \Pi=\sum_{p, q} \Pi_{p, q} e^{ \pm j \eta_{p, q} z} e^{j\left[k_{0} x \sin \theta+2 \pi\left(p x / a_{h}+q y / b_{h}\right)\right]}, \quad z \gtrless, 0
\end{aligned}
$$

where $\gamma_{p, q}$ and $\eta_{p, q}$ are non-negative defined, and the choice of the sign in the $z$ dependent exponentials corresponds to the physical requirement that the waves 
produced by the sources (21) propagate away from the plane where they lie $(z=0)$.

Letting (23) into (22), for $z \neq 0$, one readily gets:

$$
\gamma_{p, q}=\eta_{p, q}=+\sqrt{k_{0}^{2}-\left\{\left(\frac{2 \pi q}{b_{h}}\right)^{2}+\left[\left(\frac{2 \pi p}{a_{h}}\right)+k_{0} \sin \theta\right]^{2}\right\}} .
$$

Each term in (23) is thus recognized to represent a plane wave propagating in the $(p, q)$-grating lobe direction, with wave vector:

$$
k_{x}=\frac{2 \pi}{a_{h}} p+k_{0} \sin \theta, \quad k_{y}=\frac{2 \pi}{b_{h}} q, \quad k_{z}= \pm\left[k_{0}^{2}-\left(k_{x}^{2}+k_{y}^{2}\right)\right]^{1 / 2}, \quad z \gtrless 0 .
$$

In particular, the $p=q=0$ term propagates, for $x<0$, in the specular reflection direction ${ }^{8}$, with wave-vector:

$$
k_{x}=k_{0} \sin \theta, \quad k_{y}=0, \quad k_{z}=-k_{0} \cos \theta .
$$

In order to determine the coefficients $A_{p, q}$ and $\Pi_{p, q}$, we require that (23) be weaksolutions of (22), by integrating them across $(0-, 0+)$, viz.:

$$
\begin{aligned}
& j \omega \mu_{0} \int_{0-}^{0+} d z P_{t o t}=\int_{0-}^{0+} d z\left(\nabla^{2}+k_{0}^{2}\right) A= \\
& =\int_{0-}^{0+} d z\left(\partial_{x x}+\partial_{y y}+k_{0}^{2}\right) A+\left|\partial_{z} A\right|_{z=0-}^{z=0+},
\end{aligned}
$$

and the like for $\Pi$. The first term on the r.h.s. of (27) is zero, because the integrand is continuous and limited across $z=0$. The second is non-zero, because $\partial_{z} A$ is discontinuous across $z=0$, due to the different signs of the $z$-dependent exponential for $z>0$ and $z<0$ in (23). Hence, using (21) and (23) in (27) one gets:

$$
\begin{gathered}
A_{p, q}=2 \frac{k_{0} H_{0}}{a b \gamma_{p, q}} \sin \theta \mu_{0} \alpha_{e} \\
\Pi_{p, q}=-j \frac{2 H_{0}}{a b \eta_{p, q}} \alpha_{m}
\end{gathered}
$$

The reflection coefficient of the perforated wall can now be defined with reference to the specular component $(p=q=0)$ of the scattered field ${ }^{9}$ :

\footnotetext{
${ }^{8}$ Compare with $\vec{k}^{(r)}$ in $(12)$.

${ }^{9}$ In the asymptotic case of our interest, $\theta \longrightarrow \pi / 2$, all higher-order grating lobe waves $(p, q \neq 0)$ decay exponentially off the $z=0$ plane, as seen from eq. (24).
} 


$$
\Gamma_{H}=\left.\frac{H_{0,0}^{(s c a t t)}+H^{(r)}}{H^{(i)}}\right|_{z=0}:=-\frac{Z_{z>0}-Z_{z<0}}{Z_{z>0}+Z_{z<0}}
$$

where the last equality defines the reflection coefficient at $z=0$ in terms of the oblique wave impedances ${ }^{10} Z_{z<0}$ and $Z_{z>0}$ of the media filling the $z<0$ and $z>0$ half-spaces.

The scattered magnetic field can be easily computed using (19), (23) and (28), (29). One readily obtains ${ }^{11}$ :

$$
H_{0,0}^{(s c a t t .)}=2 j k_{0} \frac{\alpha_{m}+\alpha_{e} \sin ^{2} \theta}{a_{h} b_{h} \cos \theta} H_{0} e^{j \vec{k}^{(r)} \cdot \vec{r}}
$$

and hence, substituting into (30):

$$
\Gamma_{H}=1+2 j k_{0} \frac{\alpha_{m}+\alpha_{e} \sin ^{2} \theta}{a_{h} b_{h} \cos \theta} \approx 1-2 \frac{Z_{z>0}}{Z_{z<0}}=1-2 \frac{Z_{z>0}}{Z_{0} \cos \theta}
$$

where the fraction on the r.h.s. of (30) has been expanded to lowest order in the ratio $^{12} Z_{z>0} / Z_{z<0}$. Hence:

$$
Z_{z>0}=-j \frac{k_{0} Z_{0}}{a_{h} b_{h}}\left(\alpha_{m}+\alpha_{e} \sin ^{2} \theta\right) .
$$

We are thus led to conclude that, for close-to-grazing incident fields, a perforated perfectly conducting wall acts like the surface of a homogeneous medium with (oblique) wave impedance

$$
Z_{z>0}=-j \frac{k_{0} Z_{0}}{a_{h} b_{h}}\left(\alpha_{m}+\alpha_{e}\right) .
$$

Note that the spatial distribution of the holes appears in (34) only through the factor $\left(a_{h} b_{h}\right)^{-1}$ which represents the number of holes per unit area $n_{\sigma}$. Hence:

$$
Z_{z>0}=-j k_{0} Z_{0}\left(\alpha_{m}+\alpha_{e}\right) n_{\sigma}
$$

which reproduces our heuristic ansatz (8).

As a matter of fact, the impedance (35) is usually very small, and thus provided the further condition:

$$
\left|\left(\frac{Z_{0}}{Z_{z>0}}\right) k_{0} \rho_{S}\right| \gg 1
$$

\footnotetext{
${ }^{10}$ We remind that the oblique wave impedance of a plane wave is defined as the ratio between the components of $\vec{E}$ and $\vec{H}$ transverse to the direction of propagation $\vec{k}$.

${ }^{11}$ The scattered specular magnetic field has only a nonzero $y$-component.

${ }^{12}$ Note that in the limit of vanishingly small holes we get $Z_{z>0}=0$ (perfect conductor), and hence we expect $\left|Z_{z>0} / Z_{z<0}\right| \ll 1$.
} 
is satisfied (see Appendix $A$ ), where $\rho_{S}$ is the (local) smallest radius of curvature of the surface $S$, then a Leontóvich boundary condition (3) with $Z_{\text {wall }}$ given by (35), can be used even for a non-planar, perfectly conducting, perforated surface.

This provides a rigorous justification of our heuristic ansatz (8). In the following we shall denote the wall impedance of a perforated pipe wall in free space by $Z_{p . w .}^{(0)}$.

\section{Possible Model Improvements.}

It is conceivable and relatively straightforward to improve the model by, e.g.:

- i) including the effect of a non-zero wall thickness;

- ii) describing the effect of electromagnetic coupling among the holes;

- iii) taking into account the presence of a further (imperfectly conducting) tube surrounding the perforated beam pipe;

Note that all potential improvements of (35) should be gauged consistently against the omission of terms of higher order in $k \cdot$ (hole size) in the standard (Bethe's) formulae for $\alpha_{e, m}$. These terms have been discussed in [19], [20] and [21].

In this section we shall briefly review points $i$ ) to $i i i$ ) above.

\section{Holes in a Thick Wall}

A nonzero wall thickness is useful to reduce RF leakage through the pumping holes. A general formalism for computing electric and magnetic hole polarizabilities for thick walls has been set up in [22]-[24]. For circular holes one has, with good accuracy [25]:

$$
\begin{aligned}
& \left(\alpha_{e}^{(i)}+\alpha_{m}^{(i)}\right) \approx 0.57\left(\alpha_{e}^{(0)}+\alpha_{m}^{(0)}\right), \quad w / r_{0} \leq 2 \\
& \alpha_{e}^{(e)}=\frac{2}{3} r_{0}^{3} \exp \left(-\xi_{T E} w / r_{0}\right) \\
& \alpha_{m}^{(e)}=-\frac{4}{3} r_{0}^{3} \exp \left(-\xi_{T M} w / r_{0}\right),
\end{aligned}
$$

where the superfixes $(i),(e),(0)$ identify the internal, external and thin-wall polarizabilities, $r_{0}$ is the hole radius and $w$ the wall thickness, $\xi_{T E}=2.405$ and $\xi_{T M}=1.841$ are the damping constants of the dominant cut-off modes of a circular waveguide having the same radius as the holes. 


\section{Coupling Among Holes}

Electromagnetic coupling among the holes can be accounted by using in (35) the effective electric polarizabilities $\alpha_{e, m}^{\prime}$ of each hole, in the presence of the others, viz.:

$$
\alpha_{e, m}^{\prime}=\frac{\alpha_{e, m}}{1-C_{e, m} \alpha_{e, m}},
$$

where the coupling constants $C_{e, m}$ are given in Appendix B. For a relativistic beam, the induced electric dipoles are normal to the perforated wall, while the magnetic ones are tangent and, for circular holes, parallel to the magnetic field. Thus, eq.s (B5) to (B7), under the further assumption $a=b=s$ (isotropic hole array) specialize to:

$$
\begin{gathered}
C_{e}=C_{\perp}=s^{-3}\left[-\frac{12}{5 \pi}+16 \pi K_{0}(2 \pi),\right] \\
C_{m}=C_{\|}=s^{-3}\left[\frac{6}{5 \pi}-8 \pi K_{0}(2 \pi),\right]
\end{gathered}
$$

where $K_{0}$ is a Bessel function of the $3 r d$ kind. Equations (41), (42) imply a quasi static $(s \ll \lambda)$ assumption, which could be removed in principle [26].

\section{Perforated Beam Pipe in a Co-Axial Lossy Tube}

The influence of an external imperfectly conducting tube, coaxial to the beam pipe (cold-bore) can be simply described by using modified polarizabilities in eq. (7) and (8). In Appendix $C$ we show that for the special case of a circular liner of radius $b$ surrounded by a coaxial tube of radius $a$ the modified polarizabilities have the simple form:

$$
\alpha_{e, m}^{\prime}=\alpha_{e, m}^{(i)}+F \alpha_{e, m}^{(e)}
$$

where $^{13}$ :

$$
F=-\frac{\alpha_{e}^{(e)}+\alpha_{m}^{(e)}}{\alpha_{e}^{(i)}+\alpha_{m}^{(i)}+j \operatorname{sgn}(k) \hat{\delta}^{*} n_{\sigma}^{-1}(1+b / a)},
$$

or, equivalently,

$$
F=-\frac{\left(\alpha_{e}^{(e)}+\alpha_{m}^{(e)}\right)\left(\alpha_{e}^{(i)}+\alpha_{m}^{(i)}\right)^{-1}}{1+\frac{(1+b / a) Z_{c b}}{Z_{p . w .}^{(0)}}} .
$$

In (43), (44) and (45) the superfixes $(e),(i)$ denote the external and internal polarizabilities, $\hat{\delta}$ is the complex EM penetration depth into the cold bore walls

\footnotetext{
${ }^{13}$ Equation (44) was first deduced by Gluckstern [27], [28], although apparently neglecting the complex character of $\hat{\delta}$. See Appendix $C$.
} 
(both walls at $r=b+$ and $r=a$ assumed lossy, with finite conductivity $\sigma_{c b}$ ), and $Z_{c b}$ the corresponding (complex) wall impedance:

$$
\hat{\delta}=\left(\left|k_{0}\right| Z_{0}\right)^{-1} Z_{c b}^{*}, \quad Z_{c b}=[1-j \operatorname{sgn}(\omega)]\left(\frac{\left|k_{0}\right| Z_{0}}{2 \sigma_{c b}}\right)^{1 / 2} .
$$

Hence, (7) and (8) become:

$$
\begin{aligned}
& Z_{\|}=-j \frac{Z_{0} k_{0}}{2 \pi b}\left[\left(\alpha_{e}^{(i)}+\alpha_{m}^{(i)}\right)-\frac{\left(\alpha_{e}^{(e)}+\alpha_{m}^{(e)}\right)^{2}\left(\alpha_{e}^{(i)}+\alpha_{m}^{(i)}\right)^{-1}}{1+\frac{(1+b / a) Z_{c b}}{Z_{p . w .}^{(0)}}}\right] n_{\sigma}, \\
& Z_{w a l l}=-j Z_{0} k_{0} n_{\sigma}\left[\left(\alpha_{e}^{(i)}+\alpha_{m}^{(i)}\right)-\frac{\left(\alpha_{e}^{(e)}+\alpha_{m}^{(e)}\right)^{2}\left(\alpha_{e}^{(i)}+\alpha_{m}^{(i)}\right)^{-1}}{1+\frac{(1+b / a) Z_{c b}}{Z_{p . w .}^{(0)}}}\right] .
\end{aligned}
$$

In the limit of an infinitely thin liner's wall, where $\alpha_{e, m}^{(e)}=-\alpha_{e, m}^{(i)}$ equation (48) admits a simple and sound physical interpretation.

The complex propagation constant and characteristic impedance of the lossy coaxial cold bore waveguide, can be equivalently computed by assuming that the outer $(r=a)$ cold-bore wall is a perfect conductor, (as assumed from the beginning in [29]) and placing an equivalent wall impedance:

$$
Z_{e q .}=Z_{c b}\left(1+\frac{b}{a}\right)
$$

on the inner conductor $(r=b)$ alone ${ }^{14}$. Then, it is readily seen that (48) is nothing but the parallel combination of $Z_{p . w}^{(0)}$. given by eq. (8) and $Z_{e q .}$ (49),

$$
Z_{\text {wall }}=\frac{Z_{p \cdot w .}^{(0)} Z_{e q .}}{Z_{p . w .}^{(0)}+Z_{e q .}}
$$

The power lost in the coaxial region has been predicted and measured in [7], [8], and confirms the validity of the above analysis (see Appendix C.)

\section{$6 \quad$ Numerical Results}

In this section we shall apply our main results to the square geometry depicted in Fig. 4, where the holes are located in the rounded corners and the flat sides of length $d$ are copper coated.

\footnotetext{
${ }^{14}$ This is a more or less obvious consequence of the $r^{-1}$ dependance of the TEM magnetic field in the coaxial region
} 


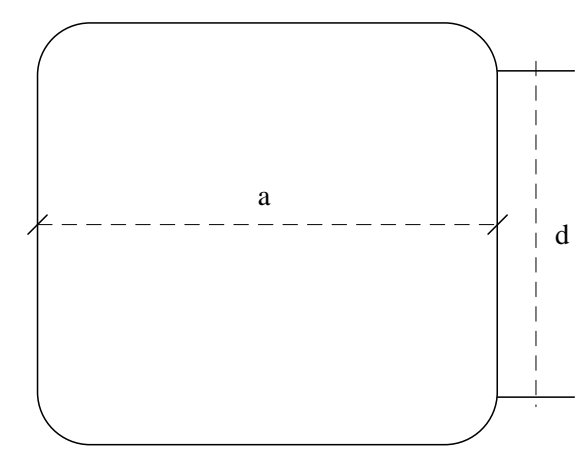

Figure 4: Square pipe with rounded corners. The radius of curvature is $(a-d) / 2$ and the flat sides are copper coated, while the corners are uncoated.

We shall assume that the size of the holes is determined by the requirement of preventing excessive radiation loss through them, while their number is dictated by requiring an adequate pumping capacity. Typical numbers are shown in Table I. If the holes are confined to the rounded corners of the square-section liner depicted in Fig. 4 , then

(no. of holes per unit length of liner) $=$ 4 (no. of holes per unit length at each rounded corner)

It is readily seen that each rounded corner has a surface $(\pi / 4)(a-d)$ per unit length of liner, thus, there are $4(\pi / 4)(a-d) n_{\sigma}$ holes per unit length of liner. Furthermore, for a regular $2 D$ lattice of circular holes with spacing $s$, one has ${ }^{15}$

$$
n_{\sigma}=s^{-2} \frac{N_{\lambda}}{4 a}\left[\frac{\pi}{4}\left(1-\frac{d}{a}\right)\right]^{-1} .
$$

As a result, the coupling coefficients (41) and (42) become functions of $d / a$ and so does the wall impedance.

Representative numerical results are displayed in Fig.s 5 and 6. In Fig. 5 we plot $R=\operatorname{Re}\left(Z_{\text {wall }}\right)$ and $X=\operatorname{Im}\left(Z_{\text {wall }}\right)$ vs. $d / a$, for $\omega / \omega_{c}=.1, .5, .9, \omega_{c}=(\pi c / a)$ being the (lowest) pipe cut-off frequency ${ }^{16}$. To draw these figures we used the parameters collected in Table I below, taking into account wall thickness via eq. (37), and the presence of the cold-bore via eq.s (43), (44).

Neglecting hole coupling, or including it via eq.s (40), (42) does not affect sensibly the curves in Fig. 5.

\footnotetext{
${ }^{15}$ Note that $s \geq 2 r_{0}$, above which the holes merge.

${ }^{16}$ Below the (lowest) cutoff frequency $(c / 2 a)$, coupling among the holes will be essentially static, consistent with (41) and (42). This can be loosely understood by noting that, in the relativistic regime, the beam field is deducible from a (transverse, scalar) potential depending on $z-c t$, and that below cut-off, no fields could propagate from one hole to another.
} 

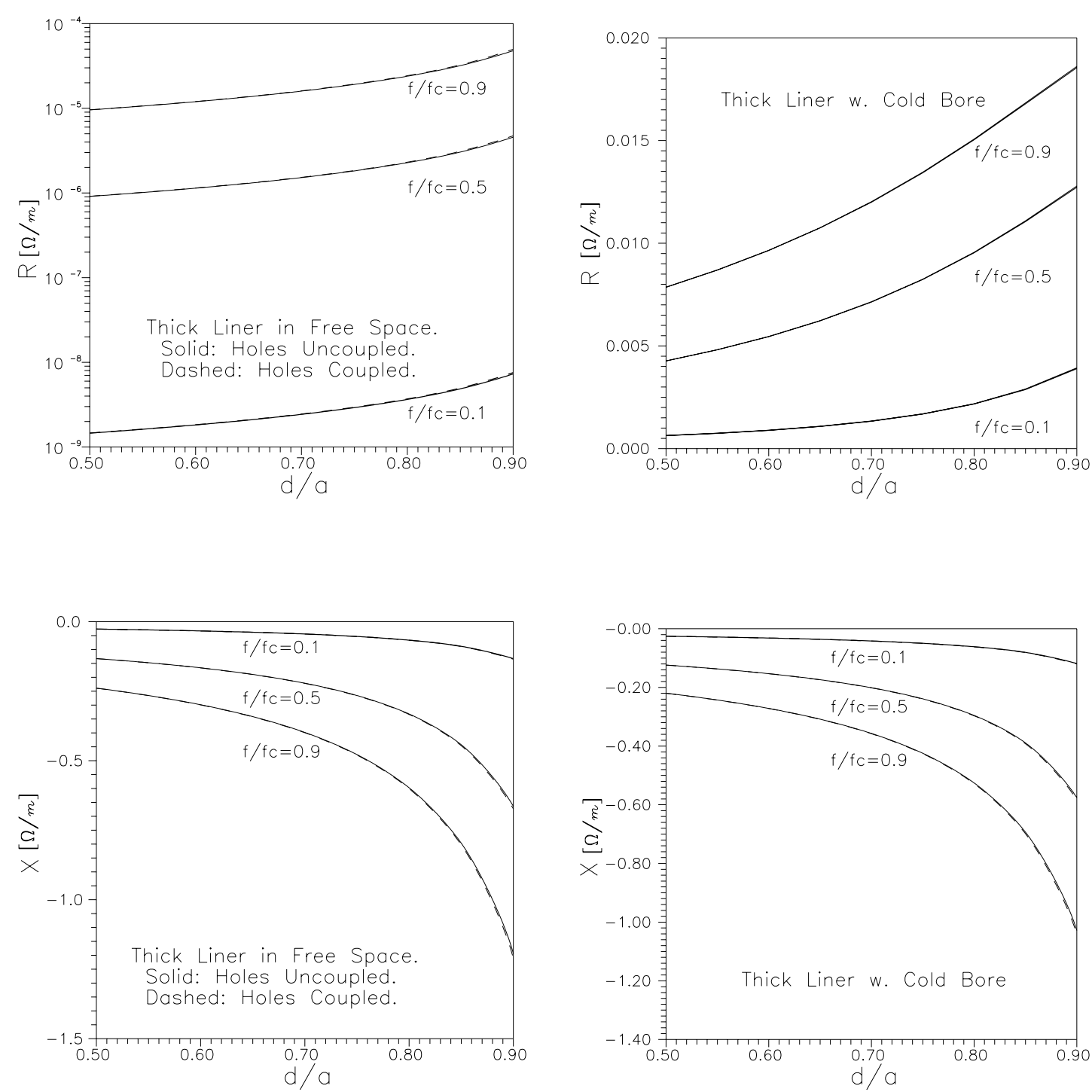

Figure 5: Thick liner in (left) free space and (right) coaxial cold bore: $R=$ $\operatorname{Re}\left(Z_{\text {wall }}\right)$ and $X=\operatorname{Im}\left(Z_{\text {wall }}\right)$ vs. $d / a$, for $\omega / \omega_{c}=.1, .5, .9, \omega_{c}=(\pi c / a)$ being the (lowest) pipe cut-off frequency. We assume the geometry of Fig. 4 with the holes confined to the rounded corners and the parameters of Table I 

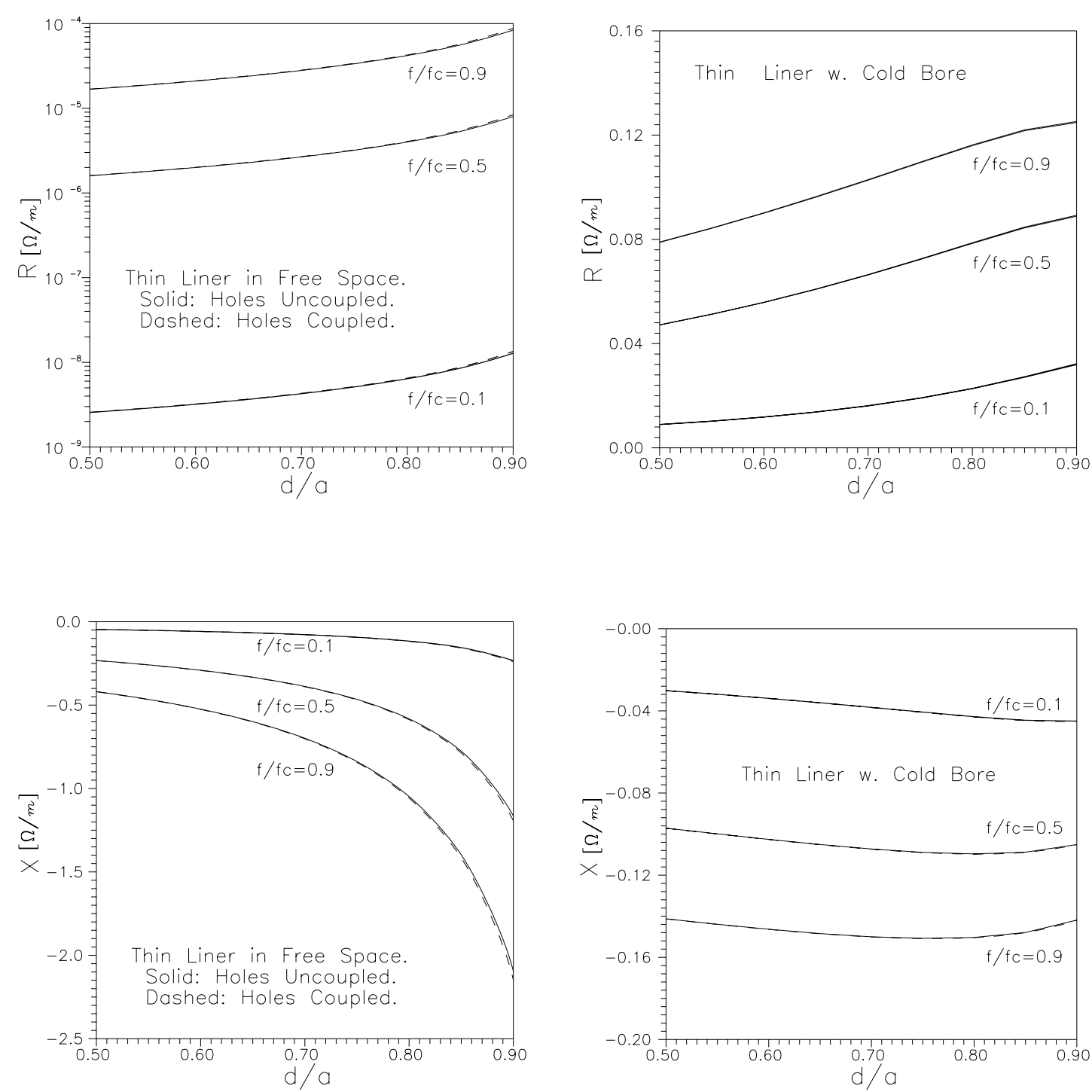

Figure 6: Thin liner in (left) free space and (right) coaxial cold bore: $R=$ $\operatorname{Re}\left(Z_{\text {wall }}\right)$ and $X=\operatorname{Im}\left(Z_{\text {wall }}\right)$ vs. $d / a$, for $\omega / \omega_{c}=.1, .5, .9, \omega_{c}=(\pi c / a)$ being the (lowest) pipe cut-off frequency. We assume the geometry of Fig. 4 with the holes confined to the rounded corners and the parameters of Table I 
On the other hand, for a thin liner in free space at low frequencies $\left(\omega \ll \omega_{c}\right)$ hole coupling can be relevant, as shown in Fig. 6, where we used Kurennoy's result:

$$
\left|\frac{\operatorname{Re} Z_{\text {wall }}}{\operatorname{Im} Z_{\text {wall }}}\right|=\frac{\pi^{2}}{6} \frac{\alpha_{e}^{2}+\alpha_{m}^{2}}{\alpha_{e}+\alpha_{m}}\left(\frac{\omega}{\omega_{c}}\right)^{3} a^{-3}
$$

and (8) together with eq.s (40), (42).

\section{Parasitic Loss Due to Resistive Wall and Holes}

The parasitic loss (energy lost by the beam per unit pipe-length), is given by [12]:

$$
\frac{\Delta \mathcal{E}}{L}=\frac{1}{2 \pi} \int_{-\infty}^{+\infty}|I(\omega)|^{2} \Re e Z_{\|}(\omega) d \omega
$$

where $I(\omega)$ is the beam current frequency spectrum, and $Z_{\|}(\omega)$ the longitudinal impedance.

In the following we shall refer to a stainless-steel, rounded-corner, square cross-section beam-pipe with copper-plated side walls, sketched in Fig. 4.

For gaussian bunches of r.m.s. length $\sigma_{z}$, and total charge $Q$, the current frequency spectrum is:

$$
I(\omega)=Q e^{-\sigma_{z}^{2} k^{2} / 2} .
$$

\subsection{Ohmic Losses}

The pure ohmic power losses $P_{C u}^{(0)}+P_{s s}^{(0)}$ in the unperforated copper-plated and stainless steel (internal) beam-pipe surfaces, can be written [34]:

$$
P_{C u, s s}^{(0)}=N_{b} \nu_{r} \frac{\Delta \mathcal{E}}{L}=N_{b} \nu_{r} \frac{Q^{2} c Z_{0}}{8 \pi^{3} a^{2}} W_{C u, s s}\left(\frac{\sigma_{z}}{a}\right) G_{C u, S S}\left(\frac{d}{a}\right),
$$

where $N_{b}$ is the number of bunches in the ring, $\nu_{r}$ the revolution frequency, $a$ the (rounded) square side-lenght, $Q$ the bunch charge, the functions $G_{C u}(\cdot), G_{S S}(\cdot)$ are defined in Appendix D, and the functions $W_{s s}(\cdot)$ and $W_{C u}(\cdot)$ depend only on the bunch length and the (local, spectral) impedances $Z_{\text {wall }}^{(\mathrm{Cu})}, Z_{\text {wall }}^{(s s)}$ of the stainless-steel and copper-plated surfaces and are [34]:

$$
W_{s s, C u}\left(\frac{\sigma_{z}}{a}\right)=2 \int_{0}^{+\infty} e^{-\left(\sigma_{z}^{2} / a^{2}\right)\left(y^{2} / \beta_{0}^{2}\right)} \Re e\left\{Y_{0} Z_{\text {wall }}^{(s s, C u)}\left(\frac{y c}{a}\right) d y\right\} .
$$

where $y=\pi \omega / \omega_{c}, \omega_{c}=\pi / a$ being the cutoff frequency of the first waveguide mode in the (square) liner, and the wall impedances $Z_{\text {wall }}^{(s s, C u)}$ refer to the unperforated (stainless steel, copper) chamber walls. In Fig. 7 we plot $W_{s s}\left(\sigma_{z} / a\right)$, using the parameters in Table I. The corresponding values of $W_{C u}\left(\sigma_{z} / a\right)$ are easily deduced since: $W_{s s} / W_{C u}=\sqrt{\rho_{s s} / \rho_{C u}} \approx 30$. 


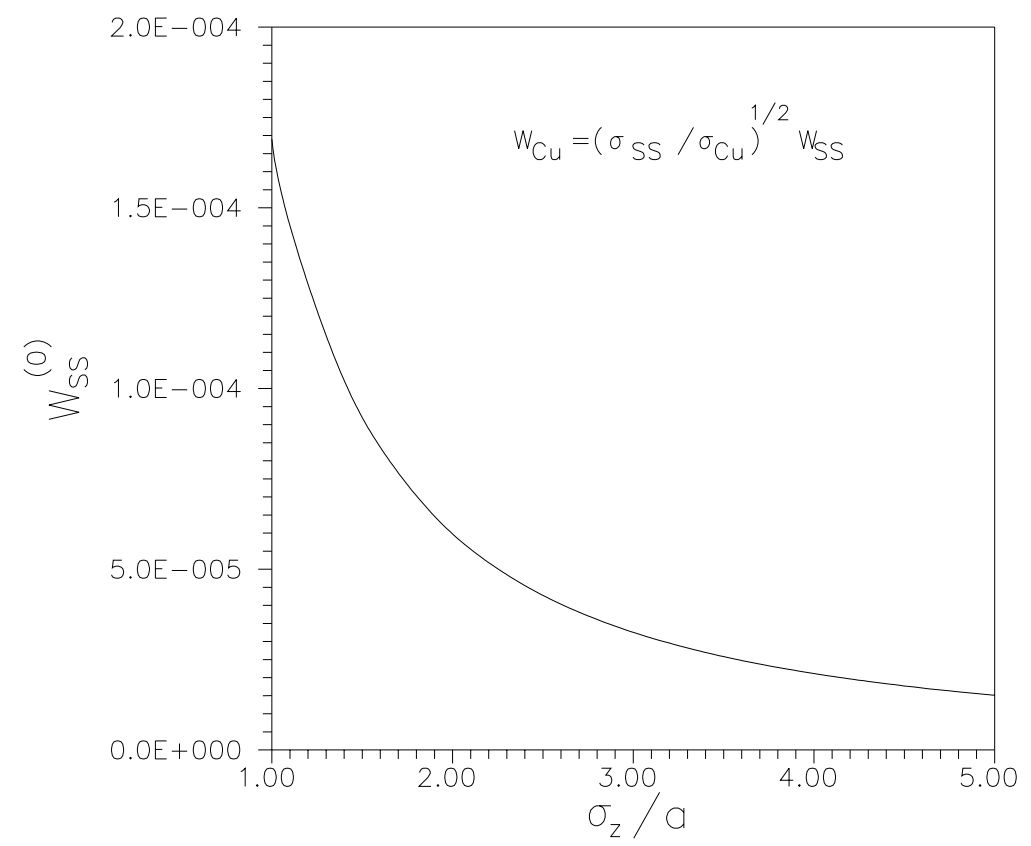

Figure 7: The function $W_{s s}\left(\sigma_{z} / a\right)$ for squared chamber geometry with unperforated walls. We assume the geometry of Fig. 4 and the parameters in Table I.

\begin{tabular}{|c|c|}
\hline \hline$\rho_{s s}$ & $5 \times 10^{-7} \Omega m$ \\
$\rho_{C u}$ & $5.5 \times 10^{-10} \Omega m$ \\
\# of particles per bunch & $10^{11}$ \\
\# of bunches $N_{b}$ & 2835 \\
revolution frequency $\nu_{r}$ & $11.245 \mathrm{kHz}$ \\
hole radius $r_{0}$ & $.75 \mathrm{~mm}$ \\
wall thickness & $.75 \mathrm{~mm}$ \\
liner diameter $a$ & $3.48 \mathrm{~cm}$ \\
bunch length $\sigma_{z}$ & $7.5 \mathrm{~cm}$ \\
\hline \hline
\end{tabular}

Table I: Model parameters. 


\subsection{Power Loss Through Holes}

The power leaking through the holes, and dissipated in the stainless-steel inner and outer walls of the coaxial region can be written as $P_{C u}^{(\text {holes })}+P_{S S}^{(\text {holes })}$, where :

$$
\begin{aligned}
P_{C u}^{(\text {holes })} & =N_{b} \nu_{r} \frac{Q^{2} c Z_{0}}{8 \pi^{3} a^{2}} W_{C u}^{(\text {holes })}\left(\frac{\sigma_{z}}{a}\right) G_{C u}\left(\frac{d}{a}\right) \\
P_{s s}^{(\text {holes })} & =N_{b} \nu_{r} \frac{Q^{2} c Z_{0}}{8 \pi^{3} a^{2}} W_{s s}^{(\text {holes })}\left(\frac{\sigma_{z}}{a}\right) G_{S S}\left(\frac{d}{a}\right)
\end{aligned}
$$

represent the contribution of the holes drilled on the stainless-steel (roundedcorners) and copper-plated (straight sides) portions of the liner's wall. In (58) and (59),

$$
W_{s s, C u}^{(\text {holes })}\left(\frac{\sigma_{z}}{a}\right)=2 \int_{0}^{+\infty} e^{-\left(\sigma_{z}^{2} / a^{2}\right)\left(y^{2} / \beta_{0}^{2}\right)} \Re e\left\{Y_{0} Z_{\text {wall }(s s, C u)}^{(\text {hols })}\left(\frac{y c}{a}\right) d y\right\} .
$$

where $Z_{\text {wall }(\text { ss, } C u)}^{(\text {ou })}$ is the appropriate wall impedance (48), which depends on the number of holes per unit surface:

$$
n_{\sigma}^{(C u)}=\frac{N_{\lambda}^{(C u)}}{4 a}\left(\frac{d}{a}\right)^{-1}, \quad n_{\sigma}^{(s s)}=\frac{N_{\lambda}^{(s s)}}{4 a}\left[\frac{\pi}{4}\left(1-\frac{d}{a}\right)\right]^{-1}
$$

$N_{\lambda}^{(C u, s s)}$ being the number of holes per unit length on the copper-plated and stainless-steel portions of the liner's wall.

The ohmic power losses in the perforated pipe can be approximately written

$$
P_{C u, s s}=\left(1-\alpha_{C u, s s}\right) P_{C u, s s}^{(0)}
$$

where $P_{C u, s s}^{(0)}$ are computed using (56) and (57), and $\alpha_{C u, s s}$ are the hole-covered copper and steel surface fractions, respectively.

In the following we shall focus on the special cases where the pumping holes are either confined to the copper-plated strips (case-I, $N_{\lambda}^{(s s)}=\alpha_{s s}=P_{s s}^{(\text {holes })}=0$ ) or to the stainless-steel rounded corners (case-II, $N_{\lambda}^{(C u)}=\alpha_{C u}=P_{C u}^{(\text {holes })}=0$ ).

In Fig. 8 we plot the functions $W_{s s, C u}^{\text {holes }}\left(\sigma_{z} / a\right)$ for different values of $d / a$, for case-I and case-II respectively ${ }^{17}$, as functions of the ratio $\sigma_{z} / a$, assuming $N_{\lambda}=$ $2660 h o l e s / m$ and circular holes of $1.5 \mathrm{~mm}$ diameter (fixed pumping capacity).

As a result, we get the values summarized in Tables II and III below:

In the limiting case where $d / a=0$ (all-steel, circular chamber) we get: $P_{S S}^{(0)} \approx$ $2.06 \mathrm{~W} / \mathrm{m}, P_{S S}^{(\text {holes })} \approx 14.4 \mathrm{~mW} / \mathrm{m}$ whereas in the other limiting case $d / a=1$ (all-copper square chamber) we obtain ${ }^{18} P_{\mathrm{Cu}}^{(0)} \approx 68 \mathrm{~mW} / \mathrm{m}, P_{\mathrm{Cu}}^{(\text {holes })} \approx 10 \mathrm{~mW} / \mathrm{m}$.

\footnotetext{
${ }^{17}$ Note that, for case-I and case-II, $d / a$ must be, respectively, larger than $\pi^{-1}\left(S_{h} N_{\lambda} / 4 a\right)$ and smaller than $1-(4 / \pi)^{-2}\left(S_{h} N_{\lambda} / 4 a\right)$, to prevent adjacent holes from merging, $S_{h}$ being the hole surface.

${ }^{18}$ Note that the quoted ohmic loss value is smaller than measured by a factor 2 . This discrepancy is probably due to the roughness of the internal chamber wall [35].
} 

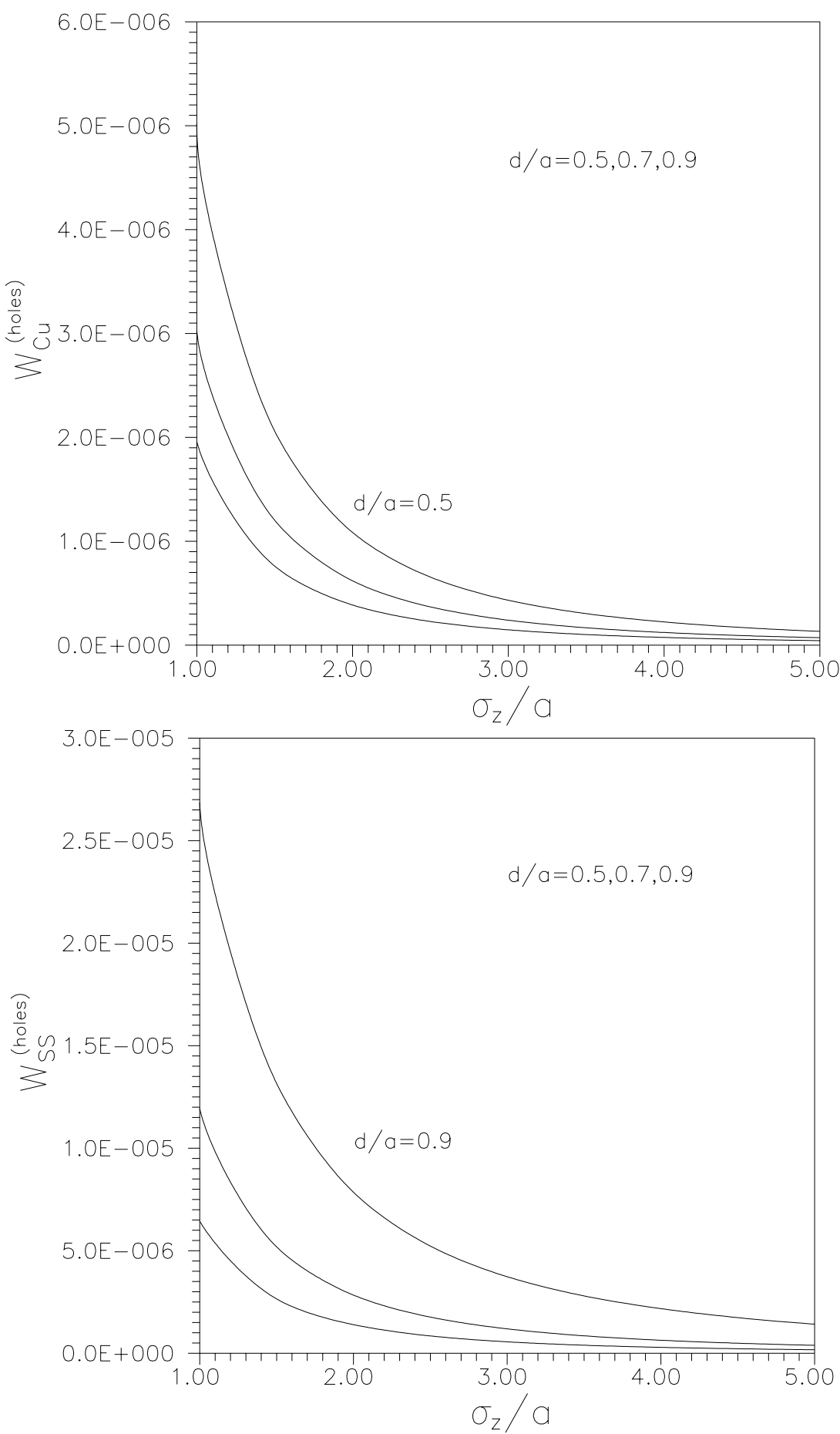

Figure 8: (Top) $W_{\mathrm{Cu}}^{\text {holes }}\left(\sigma_{z} / a\right)$ versus $\sigma_{z} / a$, holes confined to the the copper-plated strips (case I). (Bottom) $W_{s s}^{\text {holes }}\left(\sigma_{z} / a\right)$ versus $\sigma_{z} / a$, holes confined to the stainlesssteel rounded corners (case II). In both cases we assume the geometry of Fig. 4 with $d / a=.5, .7, .9$ and all other parameters as in Table I. 


\begin{tabular}{|l|c|c|}
\hline \hline & $d / a=.5$ & $d / a=.7$ \\
\hline$P_{C u}$ & $54 \mathrm{~mW} / \mathrm{m}$ & $63 \mathrm{~mW} / \mathrm{m}$ \\
$P_{S S}$ & $326 \mathrm{~mW} / \mathrm{m}$ & $72 \mathrm{~mW} / \mathrm{m}$ \\
$P_{C u}^{(\text {holes })}$ & $30 \mathrm{~mW} / \mathrm{m}$ & $19 \mathrm{~mW} / \mathrm{m}$ \\
\hline$P_{\text {total }}$ & $410 \mathrm{~mW} / \mathrm{m}$ & $154 \mathrm{~mW} / \mathrm{m}$ \\
\hline \hline
\end{tabular}

Table II: Parasitic losses for holes on copper strips (case I).

\begin{tabular}{|l|c|c|}
\hline \hline & $d / a=.5$ & $d / a=.7$ \\
\hline$P_{C u}$ & $58 \mathrm{~mW} / \mathrm{m}$ & $66 \mathrm{~mW} / \mathrm{m}$ \\
$P_{S S}$ & $298 \mathrm{~mW} / \mathrm{m}$ & $62 \mathrm{~mW} / \mathrm{m}$ \\
$P_{S S}^{(\text {holes })}$ & $7.2 \mathrm{~mW} / \mathrm{m}$ & $3.3 \mathrm{~mW} / \mathrm{m}$ \\
\hline$P_{\text {total }}$ & $363 \mathrm{~mW} / \mathrm{m}$ & $131 \mathrm{~mW} / \mathrm{m}$ \\
\hline \hline
\end{tabular}

Table III: Parasitic losses for holes on stainless-steel rounded corners (case II).

\section{Conclusions}

While relying on several simplifying assumptions (perturbative computation of beam coupling impedances and approximate boundary condition), the proposed approach includes in a remarkably simple fashion several non obvious features which are not as easily included in the standard approach.

We do believe that the combined use of reciprocity formulae (equations (1) and (2)) and impedance boundary conditions like (35) provide a powerful tool to obtain analytical estimates of the beam coupling impedances in realistic, complex and heterogeneous geometries.

As hints for future work we mention $i$ ) the possibility of deriving variational formulae for beam coupling impedances, ii) a statistical characterization of the beam coupling impedances for randomly placed holes, iii) the extension to hopefully more accurate higher order impedance boundary conditions, as discussed in [18],[30]-[33], and iv) finally the study of pipe wall roughness.

\section{Acknowledgements}

The Author wishes to thank the SL/AP group for hospitality and help during her stay at CERN, in particular Francesco Ruggiero for stimulating discussions.

\section{References}

[1] The LHC Study Group, CERN/AC/95-05 (LHC), 1995. 
[2] S.S. Kurennoy, CERN SL 91-29 (AP), 1991; Part. Acc., 39, 1, 1992; Proc. 3rd EPAC, Berlin, 1992.

[3] S.S. Kurennoy, IHEP 92-84; SSCL-636, 1993; Proc. EPAC, London, 1994; Part. Acc., 50, 167, 1995.

[4] R.L. Gluckstern, CERN SL 92-05 (AP); CERN SL 92-18 (AP); Phys. Rev. A46, 1106, 1992.

[5] S.S. Kurennoy and R.L. Gluckstern, Proc. PAC95, Dallas, USA, 1995..

[6] S. De Santis et al., Proc. EPAC-96, Sitges, Spain, 1996; Phys. Rev. E54, 800, 1996.

[7] F. Caspers, E. Jensen, F. Ruggiero, CERN/PS 92-24 (RF-AR); CERN/SL 92-15 (DI); LHC Note 186, 1992.

[8] F. Ruggiero, Part. Acc. 50, 83, 1995.

[9] M.A. Leontóvich, Issledovanya po Raspostraneniyu Radiovoln,, USSR Acad. Press, 1948.

[10] S. Petracca, Part. Acc., 50, 211, 1995.

[11] H. Bethe, Phys. Rev. 66, 163-182, 1944.

[12] A.W. Chao, Physics of Collective Beam Instabilities in High Energy Accelerators, Wiley, 1993, Sect. 2.4.

[13] G.V. Stupakov and S.S. Kurennoy, Phys. Rev. E49, 794, 1994.

[14] S.B. Cohn, IRE Proc. 39, 1416, 1952.

[15] F. de Meulenaere and J. van Bladel, IEEE Trans., AP-25, 198-205, 1977.

[16] R. de Smedt and J. van Bladel, IEEE Trans., AP-28, 703-707, 1980.

[17] S. Kurennoy, Proc. EPAC 96, Sitges, Spain, 1996, p. 871.

[18] T.B.A. Senior, IEEE Trans. AP-29, 826, 1981.

[19] J. Van Bladel, Radio Sci. 14, 319-331, 1979.

[20] Cheng et al., Proc. PAC95, Dallas, USA, 1995.

[21] A.V. Fedotov and R.L. Gluckstern, Phys. Rev. E 56, 3583-3601, 1997.

[22] N. McDonald, IEEE Trans., MTT-20, 689-695, 1972.

[23] R.L. Gluckstern and J.A. Diamond, IEEE Trans. MTT-39, 274, 1991.

[24] W.H. Cheng, et al. Proc. PAC95, Dallas (USA), 1995.

[25] B. Radak and R.L. Gluckstern, Univ. of Maryland Rept. UMDP 93-112, 1993.

[26] W.H. Eggiman, IRE Trans. MTT-9, 408, 1961.

[27] R.L. Gluckstern, CERN SL 92-06 (AP), 1992. 
[28] R.L. Gluckstern, CERN SL 92-31 (AP), 1992.

[29] R.L. Gluckstern and B.W. Zotter, CERN SL 96-56 (AP), 1996.

[30] L.N. Trefethen and L. Halpern, Math. Comp., 47, 421, 1986.

[31] J.L. Volakis and T.B.A. Senior, Proc. IEEE, 77, 5-11, 1989.

[32] M. Idemen, Proc. Phys. Soc. Japan, 59, 71, 1990.

[33] D.J. Hoppe and Y. Rahmat-Samii, J. Electr. Waves Appl., 8, 1303, 1994.

[34] S. Petracca, CERN SL 95-109 (AP), 1995.

[35] F. Caspers, M. Morvillo and F. Ruggiero, CERN LHC Project Report 115 (1997) and Proc. PAC97, Vancouver (Canada), 1997.

[36] T.B.A. Senior, Rev. Sci. Instr., B8, 419, 1960.

[37] S.M. Rytov, J. Exp. Th. Phys. USSR, 10, 120, 1940.

[38] V.A. Fock, J. Phys. USSR, 10, 13, 1946.

[39] E.L. Feinberg, Suppl. Nuovo Cimento 11, 60, 1959.

[40] J. Brown and W. Jackson, Proc. IEE, B102, 37-42, 1955.

[41] H.S. Bennett, J. Appl. Phys., 24, 785-810, 1953.

[42] R.E. Collin, Field Theory of Guided Waves, IEEE Press, 1991.

[43] I.S. Gradshteyn and I.M. Ryzhik, Table of Integrals Series and Products, Academic Press, 1980.

[44] R.F. Harrington, Time Harmonic Electromagnetic Fields, McGraw-Hill, 1966.

\section{Appendix A - Impedance Boundary Conditions}

In this section we summarize a number of less obvious issues about impedance boundary conditions. No explicit derivations are provided, but pertinent references are given.

Impedance boundary conditions were introduced and extensively studied by the Russian School, [36] - [39], and are usually credited to Leontóvich [9]. They relate the tangential electric and magnetic fields on the exterior boundary $\partial V^{-}$ of a given domain $V$, thus allowing to solve an electromagnetic boundary value problem by solving Maxwell equations in the exterior (interior) domain only. In the simplest form, they are:

$$
\left|\left(\overline{\bar{I}}-\hat{u}_{n} \hat{u}_{n}\right) \cdot \vec{E}-Z_{\text {wall }} \hat{u}_{n} \times \vec{H}\right|_{\partial V^{-}}=0,
$$

where $Z_{\text {wall }}$ is the (local) characteristic impedance of the medium in $V$, and the fields are computed at $\partial V^{-}$. 
These conditions can be applied at the surface $\partial V$ of a homogeneous, isotropic body with refractive index $n$ and smallest curvature radius or dimension $R$ provided that [39], [36]:

$$
n \gg 1, \quad \operatorname{Im}(n) k R \gg 1 .
$$

For an open surface $\partial V$ (limiting a medium of infinite extent) for which no inward normal does intersect the surface more than once, the second condition in $(A 2)$ can be relaxed into a milder one:

$$
|n| k R \gg 1 .
$$

For a flat open surface, the first condition in $(A 2)$ is sufficient. These equations admit simple physical interpretations.

For a plane open interface, the first of $(A 2)$ implies via Snell's law that the transmitted field is a plane wave, and (A1) follows from the continuity of the field components tangent to $\partial V$.

For non-planar open interfaces, eq. (A3) implies that the surface is planar at the level of the leading Fresnel zone ${ }^{19}$, which makes $(A 1)$ asympotically valid in $(|n| k R)^{-1}$.

For a compact body, or an open but re-entrant surface, the second equation in (A2) essentially ensures that the field penetration is much smaller than $R$, so that the waves are not transmitted beyond the body.

For non-homogeneous bodies, it can be shown that [37]:

$$
\begin{aligned}
& \left|\left(\overline{\bar{I}}-\hat{u}_{n} \hat{u}_{n}\right) \cdot \vec{E}-Z_{\text {wall }} \hat{u}_{n} \times \vec{H}\right|_{\partial V}= \\
= & \mathcal{O}\left(\frac{1}{k Z_{0}} \frac{\partial Z_{\text {wall }}}{\partial n}\right)+\mathcal{O}\left(\frac{1}{k Z_{0}}\left|\nabla_{t} Z_{\text {wall }}\right|\right)^{2} .
\end{aligned}
$$

Equations $(A 1)$ therefore do apply locally, provided $Z_{\text {wall }}$ is uniform over scales of the order of the wavelength in $V$. Higher order boundary conditions have been introduced by several Authors [18],[30]-[33], For locally plane stratified media, simple transmission line formulae are sufficiently accurate for all practical purposes.

\section{Appendix B - Effective Polarizability in a Plane Regular Hole Array}

In this Appendix, for the Reader's ease we summarize the approach developed by Brown [40], Bennett [41] and Collin [42] to compute the effective (electrical

\footnotetext{
${ }^{19}$ We recall that the first Fresnel zone around some point is a circle such that the distance of the observer from the center is $\lambda / 2$ less than the distance from the border.
} 
or magnetical) polarizability $\alpha^{\prime}$ of a single hole (possibly noncircular) in a plane regular array. The induced dipole moment $f$ is related to the pertinent field component by:

$$
\vec{f}=\alpha\left(\vec{F}_{0}+\vec{F}_{\text {int }}\right),
$$

where $\alpha$, is the polarizability of a single hole, $\vec{F}_{0}$, is the incident field, and $\vec{F}_{\text {int }}$ is the interaction field acting on the each and any hole due to the presence of all other holes. These latter are due to the very existence of the induced dipoles, and can thus be written:

$$
\vec{F}_{\text {int }}=C \vec{f}
$$

where $C$ depends only on the dipole orientation and the array geometry, not on the type of field (electric or magnetic).

According to eq.s $(B 1),(B 2)$, the effective polarizability is given by:

$$
\alpha^{\prime}=\frac{\alpha}{1-\alpha C}
$$

The interaction constant depends on the direction of the dipoles. It is convenient (superposition) to solve for the simplest cases where each induced dipole $f$ is parallel to one of the co-ordinate axes. For the canonical problem sketched in Fig. 2, showing a plane regular array of $y$-directed dipoles placed at $\vec{r}_{n m}=$ $\left(n a_{h}\right) \vec{u}_{x}+\left(m b_{h}\right) \vec{u}_{y}$. the interaction constant will be denoted as $C_{y}$.

The general solution of this problem, which implies no restriction about the ratio between the dipole spacing and the wavelength has been obtained by Collin ([42], prob.s 12.7 and 12.8). Here we shall confine to the simple case where a quasi-static approximation can be invoked (hole spacing $<<$ wavelength), which is appropriate for our present purposes.

The field at $\vec{r}=0$ (electrostatic or magnetostatic) due to all other dipoles at $\vec{r}_{n m} \neq 0$ can be derived from a scalar potential, viz.:

$$
\phi=\overrightarrow{u_{y}} \cdot \sum_{r_{n m \neq 0}} \frac{\vec{r}_{n m}}{4 \pi r^{3}},
$$

and the corresponding field is $F_{y}=-\partial \phi /\left.\partial y\right|_{\vec{r}=0}$. Hence, from $(B 4)$,

$$
\begin{gathered}
C_{y}=F_{y}=\frac{1}{4 \pi} \sum_{(n, m) \neq(0,0)}^{-\infty, \infty} \frac{2\left(m b_{h}\right)^{2}-\left(n a_{h}\right)^{2}}{\left[\left(m b_{h}\right)^{2}+\left(n a_{h}\right)^{2}\right]^{5 / 2}}= \\
=\frac{1}{\pi} \sum_{m=1}^{\infty}\left(m b_{h}\right)^{-3}+\frac{1}{2 \pi} \sum_{n=1}^{\infty} \sum_{m=-\infty}^{\infty} \frac{2\left(m b_{h}\right)^{2}-\left(n a_{h}\right)^{2}}{\left[\left(m b_{h}\right)^{2}+\left(n a_{h}\right)^{2}\right]^{5 / 2}}= \\
=\frac{6}{5 \pi b_{h}^{3}}-\frac{2}{\pi b} \sum_{m, n=1}^{\infty}\left(\frac{2 m \pi}{b_{h}}\right)^{2} K_{0}\left(\frac{2 m n \pi a_{h}}{b_{h}}\right) \sim
\end{gathered}
$$




$$
=\frac{6}{5 \pi b_{h}^{3}}-\frac{8 \pi}{b_{h}^{3}} K_{0}\left(\frac{2 \pi a_{h}}{b_{h}}\right),
$$

where the Poisson summation formula has been used ${ }^{20}$.

More or less obviously, if the induced dipoles were directed along the $x$-direction, one should interchange $a_{h}$ and $b_{h}$ in eq. (B5). Hence:

$$
C_{x} \approx \frac{6}{5 \pi a_{h}^{3}}-\frac{8 \pi}{a_{h}^{3}} K_{0}\left(\frac{2 \pi b_{h}}{a_{h}}\right) .
$$

Finally, for $z$-directed dipoles the interaction constant can be written as:

$$
\begin{gathered}
C_{z}=\frac{1}{4 \pi} \sum_{(n, m) \neq(0,0)}^{-\infty, \infty} \frac{\left(m b_{h}\right)^{2}+\left(n a_{h}\right)^{2}}{\left[\left(m b_{h}\right)^{2}+\left(n a_{h}\right)^{2}\right]^{5 / 2}}= \\
=-\frac{1}{4 \pi} \sum_{(n, m) \neq(0,0)}^{-\infty, \infty}\left\{\frac{2\left(m b_{h}\right)^{2}-\left(n a_{h}\right)^{2}}{\left[\left(m b_{h}\right)^{2}+\left(n a_{h}\right)^{2}\right]^{5 / 2}}+\frac{2\left(n a_{h}\right)^{2}-\left(m b_{h}\right)^{2}}{\left[\left(n a_{h}\right)^{2}+\left(m b_{h}\right)^{2}\right]^{5 / 2}}\right\}= \\
=-\left(C_{y}+C_{x}\right) .
\end{gathered}
$$

where the last equality is obtained by comparison with eq.s $(B 5),(B 6)$.

\section{Appendix C - Derivation of Equation (47).}

In the following we shall sketch the deduction of eq. (48), starting from first principles, following a route different (though equivalent) from the one in [27], and point out all approximations involved.

We work in the spectral domain $(z-c t \longrightarrow k)$, so that an $\exp (-j \omega t), \omega=\beta c k$ time-dependance is understood, different from the one usually adopted in applied electromagnetics. We assume that the spectral content of the primary field is such that only the fundamental (TEM) mode propagates in the coaxial region. The only non zero field components will be denoted by $E$ (radial electric field) and $H$

${ }^{20}$ The Poisson summation formula in its simplest form states that:

$$
\sum_{m}^{-\infty, \infty} f_{m}(\alpha)=\alpha^{-1} \sum_{m}^{-\infty, \infty} F(2 m \pi / \alpha),
$$

where $F(\cdot)$ is the Fourier transform of $f$, the equality should be intended in the $L^{2}$ spirit (i.e., almost for every $\alpha$, but on a set of zero Lebesgue measure), and the theorem is a mere consequence of Parseval spectral completeness theorem. In order to prove the formula, we only need the following result [43]:

$$
\int_{-\infty}^{\infty} e^{j k z} \frac{2 z^{2}-r^{2}}{\left(z^{2}+r^{2}\right)^{5 / 2}} d z=-2 k^{2} K_{0}(|k| r) .
$$

together with the exponentially fast decay to zero of the Bessel function $K_{0}$ with increasing argument. 
(azimuthal magnetic field). We shall assume the holes as located at $z=L, 2 L, \ldots$ so that $n_{\sigma}=(2 \pi b L)^{-1}$.

In the presence of an external co-axial tube, the elementary sources radiating into the liner will be:

$$
\left\{\begin{array}{l}
\vec{P}_{i}=\hat{r} \epsilon_{0}\left[\alpha_{e}^{(i)} E_{0}(i)+\alpha_{e}^{(e)} E_{c b}(i)\right], \\
\vec{M}_{i}=\hat{\phi}\left[\alpha_{m}^{(i)} H_{0}(i)+\alpha_{m}^{(e)} H_{c b}(i)\right], \quad i=-\infty, \ldots, \infty
\end{array}\right.
$$

where $E_{0}, H_{0}$ are the primary (forward-propagating) fields:

$$
\left\{\begin{aligned}
E_{0} & =\frac{Q}{2 \pi r} e^{j k z} \\
H_{0} & =Y_{0} E_{0}
\end{aligned}\right.
$$

$E_{c b}, H_{c b}$ are the fields in the coaxial region between the liner and the cold-bore, the argument $i$ refers to the position the $i-t h$ hole $(r=b, z=i L)$, and the superfixes $(e),(i)$ denote the external and internal hole polarizabilities.

We shall show that:

$$
E_{c b}(i)=F E_{0}(i), \quad H_{c b}(i)=F H_{0}(i),
$$

so that $(C 1)$ can be equally written:

$$
\left\{\begin{array}{l}
\vec{P}_{i}=\hat{r} \epsilon_{0}\left[\alpha_{e}^{(i)}+\alpha_{e}^{(e)} F\right] E_{0}(i) \\
\vec{M}_{i}=\hat{\phi}\left[\alpha_{m}^{(i)}+\alpha_{m}^{(e)} F\right] H_{0}(i)
\end{array}\right.
$$

which involves only the primary (unperturbed) fields, and corresponds to pretending that the liner is located in vacuo, and using the modified polarizabilities:

$$
\alpha_{e, m}^{(i)}+F \alpha_{e, m}^{(e)}
$$

for the liner's holes, to account for the effect of the coaxial region, in computing the perforated wall impedance (8), viz.:

$$
Z_{\text {wall }}=-j Z_{0} k_{0}\left[\left(\alpha_{e}^{(i)}+\alpha_{e}^{(i)}\right)+F\left(\alpha_{e}^{(e)}+\alpha_{m}^{(e)}\right)\right] n_{\sigma}
$$

In order to establish $(C 3)$, we proceed as follows.

The TEM fields radiated into the coaxial region by a single hole at $z=z_{q}=l L$, i.e., by the Bethe dipoles:

$$
\left\{\begin{array}{l}
\vec{P}_{l}=\hat{r} \epsilon_{0}\left[\alpha_{e}^{(e)} E_{0}(l)+\alpha_{e}^{(i)} E_{c b}(l)\right] \delta(z-l L), \\
\vec{M}_{l}=\hat{\phi}\left[\alpha_{m}^{(e)} H_{0}(l)+\alpha_{m}^{(i)} H_{c b}(l)\right] \delta(z-l L)
\end{array}\right.
$$

can be written: 


$$
\left\{\begin{array}{l}
E_{c b}^{(l)}(z)=\frac{C_{l}^{ \pm}}{r} e^{ \pm j k_{g}(z-l L)}, \quad z \gtrless l L, \\
H_{c b}^{(l)}(z)= \pm \frac{Y_{0} C_{l}^{ \pm}}{r} e^{ \pm j k_{g}(z-l L)}, \quad z \gtrless l L
\end{array}\right.
$$

where $k_{g}$ is the TEM propagation constant in the coaxial region, and the coefficients $C_{l}^{ \pm}$are readily evaluated by resorting to Lorentz (reciprocity) theorem in the form [42]:

$$
\begin{aligned}
& \iint_{\partial V}\left(\vec{E} \times \overrightarrow{\mathcal{H}}^{ \pm}-\overrightarrow{\mathcal{E}}^{ \pm} \times \vec{H}\right) \cdot \vec{n} d \Sigma= \\
= & j \omega \mu_{0} \overrightarrow{\mathcal{H}}^{ \pm}(l L) \cdot \vec{M}_{l}-j \omega \overrightarrow{\mathcal{E}}^{ \pm}(l L) \cdot \vec{P}_{l}
\end{aligned}
$$

where $V$ is a slice of the coaxial region limited by the planes $z=l L \pm \delta, \delta$ as small as one wishes, and $\left(\overrightarrow{\mathcal{E}}^{ \pm}, \overrightarrow{\mathcal{H}}^{ \pm}\right)$is a source-free forward or backward TEM mode. Hence:

$$
\left\{\begin{array}{l}
C_{l}^{(+)}=\frac{j k}{4 \pi b \log (a / b)}\left[\left(\alpha_{m}^{(e)}+\alpha_{e}^{(e)}\right) E_{0}(l)+\right. \\
\left.+\left(\alpha_{m}^{(i)}+\alpha_{e}^{(i)}\right) E_{c b}^{+}(l)-\left(\alpha_{m}^{(i)}-\alpha_{e}^{(i)}\right) E_{c b}^{-}(l)\right] \\
C_{l}^{(-)}=\frac{-j k}{4 \pi b \log (a / b)}\left[\left(\alpha_{m}^{(e)}-\alpha_{e}^{(e)}\right) E_{0}(l)+\right. \\
\left.+\left(\alpha_{m}^{(i)}-\alpha_{e}^{(i)}\right) E_{c b}^{+}(l)-\left(\alpha_{m}^{(i)}+\alpha_{e}^{(i)}\right) E_{c b}^{-}(l)\right]
\end{array}\right.
$$

Here the fields $E_{c b}^{( \pm)}$denote the forward and backward part of $E_{c b}$, respectively.

Following [27], we shall make the assumption that the backward fields are negligible w.r.t. the forward ones ${ }^{21}$.

Thus we shall focus on the first of $(C 10)$, neglect the last term on its r.h.s., and drop the suffix ${ }^{\prime}+{ }^{\prime}$. Using the first of $(C 10)$ and $(C 8)$, the (forward propagating) field at $r=b, z=z_{p}=p L$ due to the source at $z=z_{l}=l L$ is obtained:

$$
\begin{aligned}
E_{c b}^{(l)}(p) & =\frac{j k}{4 \pi b^{2} \log (a / b)}\left[\left(\alpha_{m}^{(e)}+\alpha_{e}^{(e)}\right) E_{0}(l)+\right. \\
& \left.+\left(\alpha_{m}^{(i)}+\alpha_{e}^{(i)}\right) E_{c b}(l)\right] e^{j k_{g}\left(z_{p}-z_{l}\right)}
\end{aligned}
$$

The total field at $z=p L$ due to all holes is obtained by summing (C11) over all $l<p$ :

$$
E_{c b}^{(t o t .)}(p)=\frac{j k}{4 \pi b^{2} \log (a / b)} \sum_{l<p}\left[\left(\alpha_{m}^{(e)}+\alpha_{e}^{(e)}\right) E_{0}(l)+\right.
$$

\footnotetext{
${ }^{21}$ The TEM waves produced by the holes accumulate coherently in the forward direction, since they travel at the same speed as the primary field of an (assumed) ultrarelativistic particle in the liner. The hole spacing is assumed to be such that no backward phasing may occur within the spectrum of the primary field.
} 


$$
\left.+\left(\alpha_{m}^{(i)}+\alpha_{e}^{(i)}\right) E_{c b}(l)\right] e^{j k_{g}\left(z_{p}-z_{l}\right)} .
$$

At this point we postulate eq. $(C 3)$, viz.:

$$
E_{c b}(p)=F E_{0}(p)
$$

and take $F$ as independent of $p$, because the (infinite) structure is invariant under the group of $z$-translations by multiples of $L$. Letting $(C 13)$ into $(C 12)$ we get:

$$
\begin{aligned}
F E_{0}(p) & =\frac{j k}{4 \pi b^{2} \log (a / b)} \sum_{l<p}\left[\left(\alpha_{m}^{(e)}+\alpha_{e}^{(e)}\right) E_{0}(l)+\right. \\
& \left.+\left(\alpha_{m}^{(i)}+\alpha_{e}^{(i)}\right) F E_{0}(l)\right] e^{j k_{g}\left(z_{p}-z_{l}\right)} .
\end{aligned}
$$

Then we use $(C 2)$ to express $E_{0}(l)$ in terms of $E_{0}(p)$, whence:

$$
\begin{gathered}
F E_{0}(p)=\frac{j k}{4 \pi b^{2} \log (a / b)} \sum_{l<p}\left[\left(\alpha_{m}^{(e)}+\alpha_{e}^{(e)}\right)+\right. \\
\left.+\left(\alpha_{m}^{(i)}+\alpha_{e}^{(i)}\right) F\right] E_{0}(p) e^{j\left(k_{g}-k\right)\left(z_{p}-z_{l}\right)} .
\end{gathered}
$$

The geometric series in $(C 15)$ is readily summed:

$$
\sum_{l<p} e^{j\left(k_{g}-k\right)(p-l) L}=\frac{1}{1-e^{j\left(k_{g}-k\right) L}} \approx \frac{-1}{j\left(k_{g}-k\right) L}
$$

where the last approximation is justified in view of the expected smallness of the exponent. Thus, from $(C 15)$,

$$
F=\frac{j k\left(\alpha_{m}^{(e)}+\alpha_{e}^{(e)}\right)}{j k\left(\alpha_{m}^{(i)}+\alpha_{e}^{(i)}\right)-4 \pi j\left(k_{g}-k\right) L b^{2} \log (a / b)} .
$$

To evaluate the difference $k_{g}-k$ in $(C 17)$, we note that the free-space wavenumber $k$ is equal to the loss-free TEM propagation constant in the coaxial region [42]. Hence $^{22}$ :

$$
k_{g}-k \approx j \frac{Z_{c b}}{Z_{0}} \frac{a+b}{2 a b \log (a / b)}
$$

\footnotetext{
${ }^{22}$ Equation $(C 16)$ follows immediately using the following 1 st-order formula for the (longitudinal) wavenumber in perturbed waveguides whose walls are described by an impedance boundary condition [44]:

$$
k_{\text {pert. }}-k_{\text {unpert. }}=j \frac{\int_{\partial S} Z_{c b}\left|\vec{H}_{0}\right|^{2} d \ell}{2 \operatorname{Re} \int_{S} \vec{E}_{0}^{*} \times \vec{H}_{0} \cdot \hat{n} d S},
$$

where $S$ is the cross-section, $\partial S$ its contour, $\left(E_{0}, H_{0}\right)$ the unperturbed mode, and $Z_{c b}$ is the complex (cold-bore) wall characteristic impedance.
} 
Substitution of $(C 18)$ into $(C 17)$ gives after a little algebra (no further approximations involved):

$$
F=-\frac{\left(\alpha_{e}^{(e)}+\alpha_{m}^{(e)}\right)\left(\alpha_{e}^{(i)}+\alpha_{m}^{(i)}\right)^{-1}}{1+\frac{(1+b / a) Z_{c b}}{Z_{p . w .}^{(0)}}}
$$

where $Z_{p . w}^{(0)}$. is the wall impedance of the perforated wall in free space, given by eq. (8). Equation $(C 19)$ can also be written:

$$
F=-\frac{\alpha_{e}^{(e)}+\alpha_{m}^{(e)}}{\alpha_{e}^{(i)}+\alpha_{m}^{(i)}+j \operatorname{sgn}(k) \hat{\delta}^{*} n_{\sigma}^{-1}(1+b / a)},
$$

where $\hat{\delta}$ is the complex EM penetration depth into the lossy coax-walls, viz.:

$$
\hat{\delta}=\left(\left|k_{0}\right| Z_{0}\right)^{-1} Z_{\text {wall }}^{*}, \quad Z_{\text {wall }}=[1-j \operatorname{sgn}(\omega)]\left(\frac{\left|k_{0}\right| Z_{0}}{2 \sigma_{c b}}\right)^{1 / 2} .
$$

Note that in [27], [28] the complex character of $(C 16)$ is apparently ignored.

The parasitic losses in the co-axial region have been computed in [7] in terms of the co-axial transmission line current $I_{c b}$. In view of the relationship between this latter and the magnetic field in the co-axial region, using eq.s $(C 2),(C 3)$, $(C 13)$ one gets (for a pointlike bunch $I(z, t)=Q \beta c \delta(z-\beta c t)$ ):

$$
\left|\frac{I_{c b}(z, \omega)}{I(z, \omega)}\right|=|F| \text {. }
$$

Remarkably, the result obtained in [7] using a different approach is recovered, provided

$$
\left|\alpha_{e}^{(i)}+\alpha_{m}^{(i)}\right| \ll\left|n_{\sigma}^{-1} \hat{\delta}(1+b / a)\right|,
$$

which holds true for the case discussed in [7].

\section{Appendix D - The Functions $G_{C u, S S}(d / a)$.}

The general formula (1) can be applied to estimate the real part of the longitudinal impedance of a possible LHC liner, sketched in Fig. 4, using the exact solution $E_{0}$ for the field produced by a relativistic unit-charge particle traveling along the axis of a perfectly conducting square-section pipe obtained in [34], yielding:

$$
\Re e\left[Z_{\|}(\omega)\right]=\frac{\epsilon_{0}}{\beta_{0} c Q^{2}} Y_{0} \oint_{\partial S} Z_{w a l l}\left|E_{0 n}(\vec{r}, 0)\right|^{2} d \ell .
$$

For the geometry of Fig. 4, the pipe wall cross-section contour $\partial S$, can be written as $\partial S_{S S}+\partial S_{C u}$, the first term representing the stainless-steel rounded 


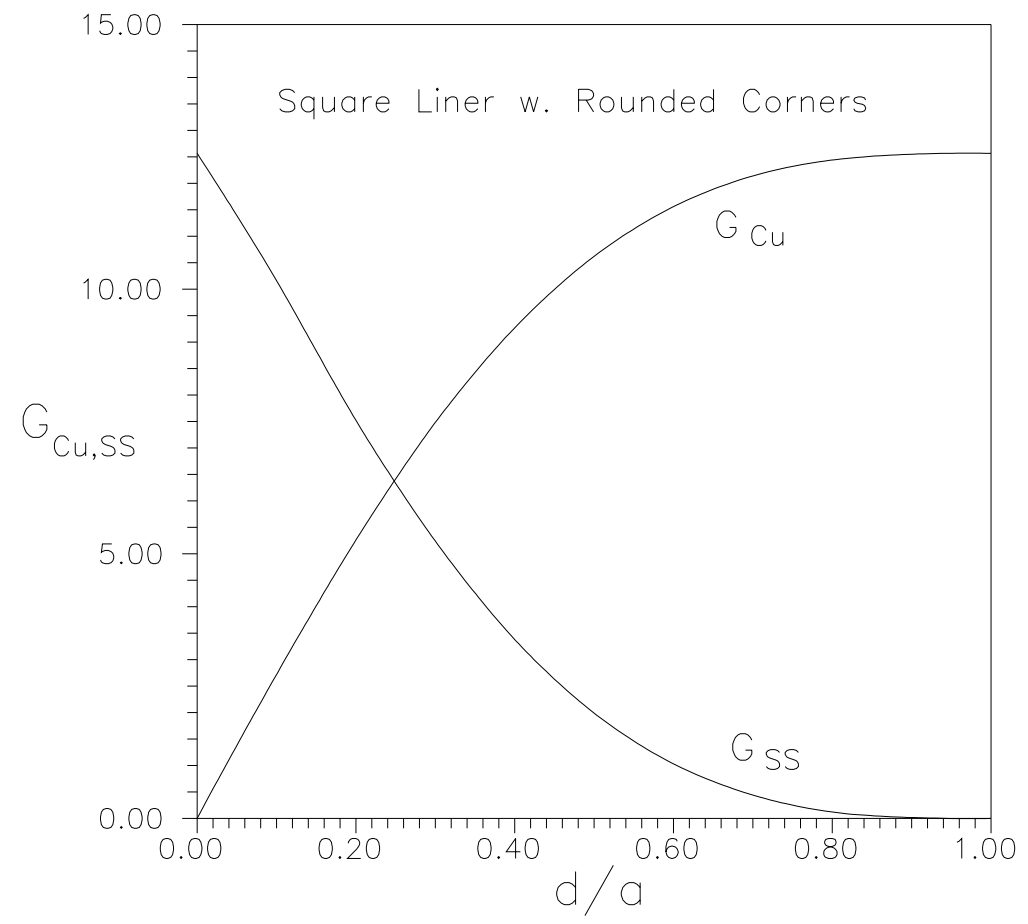

Figure 9: The functions $G C u, S S(d / a)$ for the geometry of Fig. 4.

corners, the second one the copper plated flat sides. Thus, eq. (D1) can be further written:

$$
\Re e\left[Z_{\|}(\omega)\right]=\frac{Z_{\text {wall }}^{(s s)}}{4 \pi^{2} a} G_{S S}(d / a)+\frac{Z_{\text {wall }}^{(C u)}}{4 \pi^{2} a} G_{C u}(d / a),
$$

where $Z_{\text {wall }}^{(s s)}, Z_{\text {wall }}^{(\mathrm{Cu})}$ are the wall impedances for the unperforated stainless steel and copper plated) chamber walls, respectively, and

$$
G_{C u, S S}=\oint_{\partial S_{C u, S S}}\left|\mathcal{E}_{n}(\vec{r}, 0)\right|^{2} \frac{d \ell}{a},
$$

with

$$
\overrightarrow{\mathcal{E}}(\vec{r}, 0)=\left(2 \pi \epsilon_{0} a\right) \vec{E}_{0}(\vec{r}, 0) .
$$

The functions $G_{C u, S S}$ are shown in Fig. 9 below. 